\title{
O idoso portador de Alzheimer e o cuidador familiar: contribuições da teoria de
} alcance de metas de Imogene King

\author{
Alzheimer's elderly carrier and family caregiver: contributions from Imogene King's goal \\ achievement theory \\ El anciano con Alzheimer y el cuidador familiar: contribuciones de la teoría del alcance de la meta \\ de Imogene King
}

Any Karoliny Soares Bezerra ORCID: https://orcid.org/0000-0003-0500-6359 Universidade Tiradentes, Brasil

E-mail: Karol.1997.sb@outlook.com

Eduarda Karolyne Silva Bezerra ORCID: https://orcid.org/0000-0002-1272-2394 Universidade Tiradentes, Brasil

E-mail: eduardabezerra@outlook.com

Emília Cervino Nogueira

ORCID: https://orcid.org/0000-0002-5035-6596 Universidade Tiradentes, Brasil

E-mail: emiliacervinonogueira@gmail.com

Jussiana Penha da Silva Almeida ORCID: https://orcid.org/0000-0002-1125-8678 Universidade Tiradentes, Brasil

E-mail: jussiavapenha@gmail.com Letícia de Souza Ramos

ORCID: https://orcid.org/0000-0003-0884-8682 Universidade Tiradentes, Brasil E-mail: Leticia.novolhotmail.com

Manuela de Carvalho Vieira Martins ORCID: https://orcid.org/0000-0003-1222-5955 Universidade Tiradentes, Brasil

E-mail: manuela.cvm@hotmail.com

\begin{abstract}
Resumo
Objetivo: Refletir sobre a contribuição da Teoria do Alcance de Metas de Imogene King na construção de uma assistência efetiva ao cuidador familiar do idoso portador de Alzheimer. Metodologia: Revisão integrativa de literatura realizada nas bases de dados: SCIELO, BVS, LILACS e Medline/Pubmed a partir de estudos disponíveis em inglês, português e espanhol, publicados entre 2016 e 2021. Resultados: Foram selecionados 19 artigos que possuíam relevância para esta revisão. Entre os sintomas neuropsiquiátricos apresentados pelos idosos, apatia, ansiedade e depressão são os mais frequentes. Estudos também apontam perda de memória, linguagem, funções cognitivas, controle emocional, social e perda da motivação. Os cuidadores de idosos com Alzheimer necessitam de suporte profissional de saúde, organização da família, atenção médica pessoal e apoio emocional. É importante incentivar o bem-estar próprio do cuidador para garantir e melhorar o acesso aos cuidados de saúde, prevenir o declínio funcional desses cuidadores, oferecendo orientações sobre o cuidado de maneira adequada e com menor risco. Devem-se utilizar tecnologias de cuidado adaptadas para o ambiente domiciliar e gerontotecnologias que contribuem diretamente no cuidado, assim promovendo um cuidado informativo e educativo. Conclusão: $\mathrm{O}$ cuidador familiar é aquele que possui o maior contato com o idoso portador de Alzheimer. Neste sentido, a Teoria do Alcance de Metas proposta por Imogene King permite compreender sobre o impacto do cuidado ao paciente portador de Alzheimer no cuidador familiar, suas necessidades pessoais, bem como a estratégias que podem ser utilizadas a fim se alcançar as metas estabelecidas no apoio a esse cuidador.
\end{abstract}

Palavras-chave: Cuidadores; Doença de Alzheimer; Saúde do idoso; Teoria de enfermagem.

\footnotetext{
Abstract

Objective: To reflect on the contribution of Imogene King's Goal Reach Theory in the construction of effective assistance to family caregivers of elderly patients with Alzheimer's. Methodology: Integrative literature review carried out in the following databases: SCIELO, BVS, LILACS and Medline/Pubmed based on studies available in English, Portuguese and Spanish, published between 2016 and 2021. Results: 19 articles that were relevant to this study were
} 
selected. revision. Among the neuropsychiatric symptoms presented by the elderly, apathy, anxiety and depression are the most frequent. Studies also point to loss of memory, language, cognitive functions, emotional and social control and loss of motivation. Caregivers of elderly people with Alzheimer's need professional health support, family organization, personal medical attention, and emotional support. It is important to encourage the caregiver's own wellbeing in order to guarantee and improve access to health care, prevent the functional decline of these caregivers, offering guidance on adequate care and with less risk. Care technologies adapted to the home environment and gerontotechnologies that directly contribute to care must be used, thus promoting informative and educational care. Conclusion: The family caregiver is the one who has the greatest contact with the elderly with Alzheimer's. In this sense, the Goal Attainment Theory proposed by Imogene King allows us to understand the impact of caring for patients with Alzheimer's on the family caregiver, their personal needs, as well as the strategies that can be used in order to achieve the goals established in the support to that caregiver.

Keywords: Caregivers; Alzheimer's disease; Elderly health; Nursing theory.

\section{Resumen}

Objetivo: Reflexionar sobre el aporte de la Teoría Goal Reach de Imogene King en la construcción de una asistencia eficaz a los cuidadores familiares de pacientes ancianos con Alzheimer. Metodología: Revisión integrativa de la literatura realizada en las siguientes bases de datos: SCIELO, BVS, LILACS y Medline / Pubmed en base a estudios disponibles en inglés, portugués y español, publicados entre 2016 y 2021 . Resultados: Se seleccionaron 19 artículos relevantes para este estudio. revisión. Entre los síntomas neuropsiquiátricos que presentan los ancianos, la apatía, la ansiedad y la depresión son los más frecuentes. Los estudios también apuntan a la pérdida de memoria, lenguaje, funciones cognitivas, control emocional y social y pérdida de motivación. Los cuidadores de personas mayores con Alzheimer necesitan apoyo de salud profesional, organización familiar, atención médica personal y apoyo emocional. Es importante incentivar el propio bienestar del cuidador con el fin de garantizar y mejorar el acceso a la atención de la salud, prevenir el deterioro funcional de estos cuidadores, ofreciendo orientación sobre una atención adecuada y con menor riesgo. Se deben utilizar tecnologías de cuidado adaptadas al entorno del hogar y gerontotecnologías que contribuyan directamente al cuidado, promoviendo así el cuidado informativo y educativo. Conclusión: El cuidador familiar es quien tiene mayor contacto con los ancianos con Alzheimer. En este sentido, la Teoría de la Consecución de Objetivos propuesta por Imogene King nos permite comprender el impacto del cuidado de los pacientes con Alzheimer en el cuidador familiar, sus necesidades personales, así como las estrategias que se pueden utilizar para alcanzar las metas establecidas en el apoyo a ese cuidador.

Palabras clave: Cuidadores; Enfermedad de Alzheimer; Salud de los ancianos; Teoría de enfermería.

\section{Introdução}

O envelhecimento populacional está altamente ligado à queda nas taxas demortalidade e fecundidade. Atualmente, pessoas maiores de 60 anos estão em grande maioria em relação às crianças menores de 14 anos, correspondendo respectivamente a $22,1 \%$ e $19,6 \%$ da população mundial. Segundo o Relatório Mundial de Envelhecimento e Saúde de 2015, a população com idade acima de 60 anos cresce além da média mundial e estima-se que, em 2050, os idosos irão passar de 24,4 milhões para quase 70 milhões (Dadalto et al., 2021).

No cenário brasileiro, $85 \%$ dos idosos possuem pelo menos uma doença crônica e 10\% possuem comorbidade; entre as doenças crônicas não transmissíveis (DCNTs) estão às demências, reconhecendo-se como maior problema de saúde na população idosa, particularmente na faixa etária de 80 anos (Santana et al., 2020).

A demência é o declínio geral das habilidades mentais, podendo gerar dependência e danos não só para aqueles que sofrem da doença, mas também para os seus cuidadores e familiares. Apesar de ser mais frequentemente associada ao envelhecimento entre os idosos, não é uma consequência normal. Na maioria dos países ainda existem estigmas sobre essas doenças, o que gera barreiras ao diagnóstico e ao cuidado (Mendes et al., 2016).

Ainda de acordo com Mendes et al. (2016), a Doença de Alzheimer é considerada a forma mais comum de demência neurodegenerativa em pessoas idosas, trazendo prejuízos nas habilidades cognitivas, como memória, comportamento, pensamento, compreensão, linguagem e são comumente acompanhadas pela perda de controle emocional. A perda dessas funções está ligada a atividades de vida diária ou o reconhecimento de pessoas e lugares, causando incapacidade e dependência do idoso, assim afetando a vida não apenas do idoso portador da doença como também a de seus familiares e cuidadores.

O cuidador de uma pessoa com Alzheimer deve ser preparado para observar e agir de acordo com os sintomas da doença. O cuidar é considerado como uma das tarefas mais árduas, já que o cuidador irá acompanhar o idoso em todas as 
atividades diárias, o que provoca uma modificação em sua vida, podendo ocasionar sobrecarga e trazer consequências para sua saúde psicológica e física. Dessa forma, destaca-se a importância da família no processo do cuidar a esse idoso, uma vez que na maioria dos casos os familiares são os principais cuidadores de idosos com Alzheimer (Oliveira et al., 2020).

Os mesmos autores reiteram que o cuidador familiar é submetido a uma jornada diária assídua, repetitiva, já que o idoso requer um cuidado contínuo. Muitos desses cuidadores assumem o papel sozinho, sem ajuda de terceiros. Consequentemente sua vida social muda bastante devido à progressão da patologia, e muitos podem apresentar dificuldades para compreenderem essas mudanças. Toda a sobrecarga pode gerar altos níveis de estresse, depressão e sofrimento para o cuidador familiar, e acarretar doenças agudas e crônicas. Dessa forma, faz-se necessário um olhar atento não só para as pessoas com Alzheimer, mas também seus cuidadores (Oliveira et al., 2020).

Ao tratar-se do cuidado, as teorias de enfermagem surgem como instrumento para construção do cuidado, estruturação profissional e reconhecimento social, englobando atos, comportamentos e atitudes que auxiliam na execução do cuidar. Entretanto existem maneiras distintas de cuidar, que variam de acordo com as condições em que ocorrem e o tipo de relacionamento estabelecido. Por isso torna-se necessário a utilização das teorias para desenvolver uma assistência humanizada (Silva et al., 2018).

Deste modo, Imogene King propõe a Teoria do Alcance de Metas, a qual infere que através da relação entre paciente e enfermeiro, é possível estabelecer em conjunto àsnecessidades individuais de cada pessoa, tendo como objetivo obter os melhores resultados apartir da definição de metas. Tal teoria não se restringe a apenas uma pessoa, podendo estender-se a um grupo social com o qual o enfermeiro tem contato (Pissinati et al., 2020). O presente estudo tem como objetivo geral refletir sobre a contribuição da Teoria do Alcance de Metas de Imogene King na construção de uma assistência efetiva ao cuidadorfamiliar do idoso portador de Alzheimer. Tem-se como objetivos específicos: Identificar aspectos do comportamento do idoso portador de Doença de Alzheimer; Relatar necessidadesdo cuidador familiar do idoso com Doença de Alzheimer à luz da Teoria de King; Descrevermetas a serem estabelecidas na prestação de assistência ao cuidador familiar de idoso com Alzheimer.

\section{Metodologia}

Este estudo baseou-se em uma estratégia de revisão integrativa de literatura, de caráter exploratório, para construção de uma análise ampla da literatura, tendo como ponto de partida a questão norteadora: Qual a contribuição da Teoria de Alcance de Metas de Imogene King na construção de uma assistência efetiva ao cuidador/familiar do idoso portador de Alzheimer?

Para seleção dos artigos, utilizaram-se as seguintes bases de dados: Scientific Eletronic Library Online (SCIELO), Biblioteca Virtual de Saúde (BVS), Literatura Latino-Americana e do Caribe em Ciências da Saúde (LILACS) e Medline/Pubmed, por meio das seguintespalavras chaves: Teoria de enfermagem, Doença de Alzheimer, Cuidadores e Saúde do Idoso, validados pelos Descritores em Ciências da Saúde (DeCS), utilizando o operador booleano "AND".

A análise dos dados bibliográficos foi realizada entre Agosto e Setembro de 2021, sendo selecionados 19 estudos. Foram considerados como critério de inclusão: estudos disponíveis nos idiomas inglês, português e espanhol, nas bases de dados citados e publicados entre 2016 e 2021. Os critérios de exclusão foram: artigos que geravam custos, duplicados nasbases de dados, além dos que não abordavam a temática proposta.

Para realização deste estudo, considerou-se como ponto de partida: Identificação do tema e elaboração da pergunta norteadora; Busca e seleção dos artigos científicos nas bases de dados, estabelecendo os critérios de inclusão e exclusão; Categorização das informações que foram extraídas dos artigos selecionados; Análise de dados incluídos na revisão; Discussãodos resultados da pesquisa; Apresentação da síntese do conhecimento da revisão integrativa. 
Para esta pesquisa, não se fez necessário o julgamento do Comitê de Ética em Pesquisa com seres humanos, ainda assim, será desenvolvida em conformidade com as diretrizes e normas regulamentadoras da Resolução 510/2016 do Conselho Nacional de Saúde.

\section{Resultados e Discussão}

Foram encontrados 61 artigos nas bases de dados para a realização do presente estudo. Destes, 10 foram excluídos por estarem duplicados nas bases de dados, quatro após leitura do título, 25 após leitura do resumo, dois por gerar custos e um devido ao tempo de publicação ser superior a cinco anos. Foram então selecionados 19 artigos que possuíam relevância para esta revisão (Quadro 1), dos quais, 12 encontravam-se na base de dados Scientific EletronicLibrary Online (SCIELO) e sete na Literatura Latino-Americana e do Caribe em Ciências da Saúde (LILACS). As publicações encontradas na base de dados Medline/Pubmed forameliminadas após filtro dos critérios de exclusão (Figura 1).

Figura 1 - Fluxograma de seleção dos artigos elegíveis.

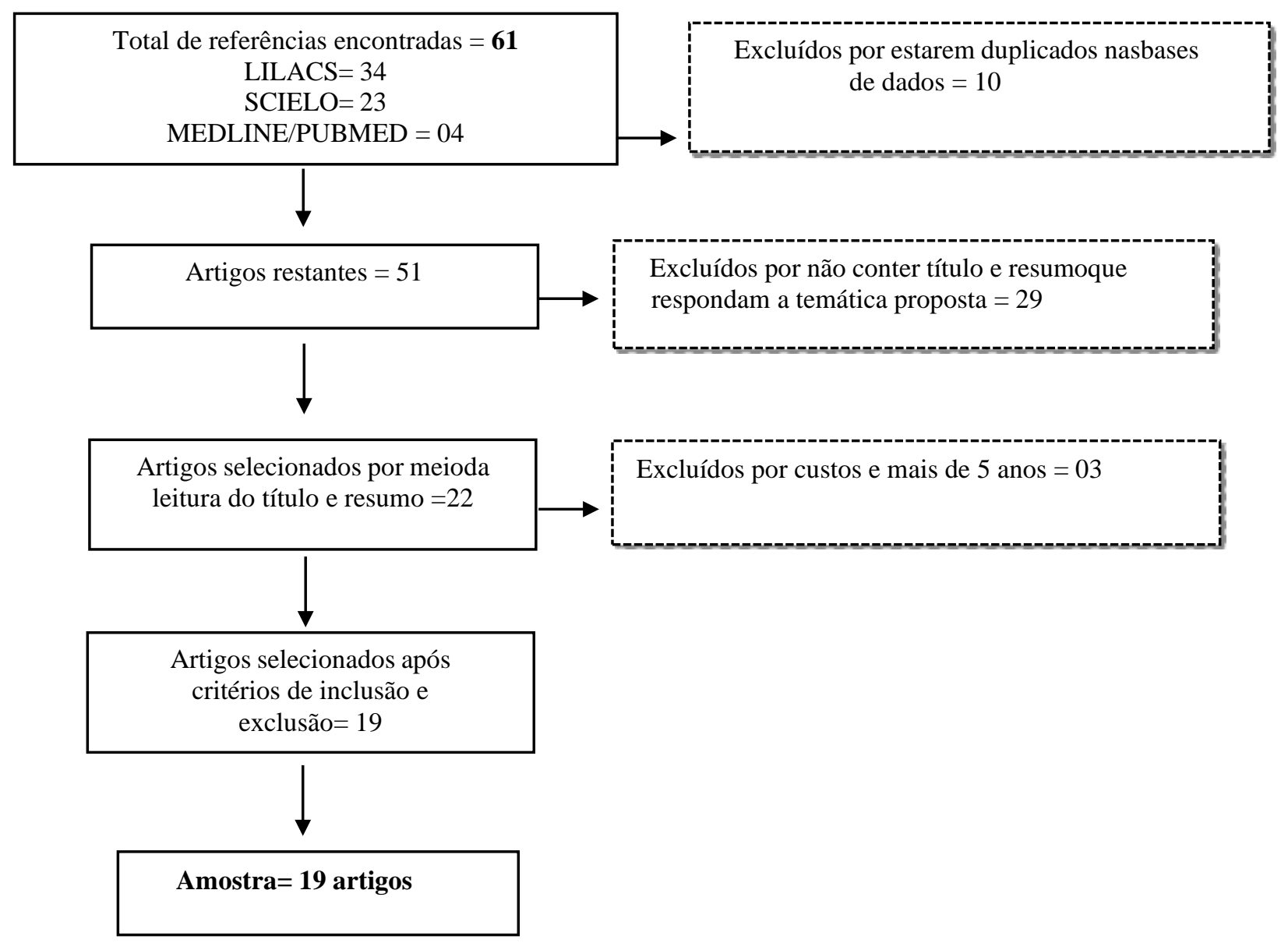

Fonte: Autoria própria (2021). 
Quadro 1 - Levantamento bibliográfico dos artigos de relevância para o estudo, 2021.

\begin{tabular}{|c|c|c|c|c|c|c|c|c|}
\hline & $\begin{array}{c}\text { BASE } \\
\text { DE } \\
\text { DADOS }\end{array}$ & TÍTULO & ANO & $\begin{array}{l}\text { AUTOR } \\
\text { (ES) }\end{array}$ & $\begin{array}{l}\text { TIPO DE } \\
\text { ESTUDO }\end{array}$ & $\begin{array}{c}\text { ASPECTOS DO } \\
\text { COMPORTAMENTO DO } \\
\text { IDOSO }\end{array}$ & $\begin{array}{c}\text { NECESSIDADES } \\
\text { DO CUIDADOR } \\
\text { FAMILIAR } \\
\end{array}$ & $\begin{array}{c}\text { METAS NA } \\
\text { PRESTAČ̃̃̃ DA } \\
\text { ASSISTENCIA }\end{array}$ \\
\hline 1 & Scielo & $\begin{array}{c}\text { Sintomas } \\
\text { neuropsiquiátric } \\
\text { os associados à } \\
\text { sobrecarga e } \\
\text { depressão do } \\
\text { cuidador } \\
\text { familiar. }\end{array}$ & 2021 & $\begin{array}{l}\text { Delfino, } \\
\text { Laís Lopes } \\
\text { et al. }\end{array}$ & $\begin{array}{l}\text { Exploratório } \\
\text { de campo }\end{array}$ & $\begin{array}{l}\text { Apatia, ansiedade e depressão são } \\
\text { os sintomas neuropsiquiátricos } \\
\text { mais frequentes. Dos } 12 \text { sintomas } \\
\text { neuropsiquiátricos investigados, } \\
10 \text { associaram-se } \\
\text { significativamente à sobrecarga } \\
\text { do cuidador (exceto ansiedade e } \\
\text { alteração alimentar), e oito } \\
\text { sintomas neuropsiquiátricos } \\
\text { apresentaram correlações } \\
\text { significativas com os sintomas de } \\
\text { depressão. }\end{array}$ & $\begin{array}{l}\text { O cuidador deve } \\
\text { receber cuidados e } \\
\text { orientação da equipe } \\
\text { de saúde para que } \\
\text { possa continuar } \\
\text { desempenhando sua } \\
\text { função com qualidade. }\end{array}$ & $\begin{array}{c}\text { Continuar } \\
\text { desempenhando sua } \\
\text { função com qualidade. }\end{array}$ \\
\hline 2 & Scielo & $\begin{array}{l}\text { O lugar do } \\
\text { cuidador } \\
\text { familiar de } \\
\text { idosos com } \\
\text { doença de } \\
\text { Alzheimer: uma } \\
\text { revisão de } \\
\text { literatura no } \\
\text { Brasil e Estados } \\
\text { Unidos. }\end{array}$ & 2021 & $\begin{array}{l}\text { Dadalto, } \\
\text { Eliane } \\
\text { Varanda et } \\
\text { al. }\end{array}$ & $\begin{array}{l}\text { Revisão de } \\
\text { literatura }\end{array}$ & & $\begin{array}{l}\text { É preciso reunir várias } \\
\text { estratégias terapêuticas } \\
\text { e farmacológicas, } \\
\text { psicoterapias, } \\
\text { atividades educativas, } \\
\text { informativas e } \\
\text { didáticas, capacitação } \\
\text { de competências } \\
\text { pessoais e sociais, } \\
\text { encontros por meio de } \\
\text { grupos focais, em que } \\
\text { haja a possibilidade de } \\
\text { acompanhamento } \\
\text { sistemático e } \\
\text { longitudinal. }\end{array}$ & $\begin{array}{l}\text { Ressaltar que quanto } \\
\text { mais grave o quadro } \\
\text { demencial, mais } \\
\text { monitoramento deve } \\
\text { haver entre o cuidador } \\
\text { primário e o secundário, } \\
\text { acionando } \\
\text { o suporte de redes de } \\
\text { atenção à saúde, de } \\
\text { atenção primária e de } \\
\text { serviços. }\end{array}$ \\
\hline 3 & Lilacs & $\begin{array}{l}\text { Telecuidado no } \\
\text { acompanhament } \\
\text { o pós-alta de } \\
\text { idosos com } \\
\text { demência e seus } \\
\text { cuidadores: } \\
\text { quase- } \\
\text { Experimental. }\end{array}$ & 2020 & $\begin{array}{l}\text { Santana, } \\
\text { Rosimeire } \\
\text { Ferreira et } \\
\quad \text { al. }\end{array}$ & $\begin{array}{l}\text { Quantitativa, } \\
\text { quase- } \\
\text { experimental }\end{array}$ & & & $\begin{array}{l}\text { Incentivar o Telecuidado } \\
\text { no cuidador de } \\
\text { enfermagem pós-alta } \\
\text { para garantir e melhorar } \\
\text { o acesso aos cuidados de } \\
\text { saúde. }\end{array}$ \\
\hline 4 & Lilacs & $\begin{array}{c}\text { Fatores } \\
\text { associados à } \\
\text { qualidade de } \\
\text { vida na } \\
\text { perspectiva do } \\
\text { Cuidador de } \\
\text { idoso com a } \\
\text { doença de } \\
\text { Alzheimer. }\end{array}$ & 2020 & $\begin{array}{l}\text { Damásio, } \\
\text { Clélia } \\
\text { Santana } \\
\text { Reis et al. }\end{array}$ & $\begin{array}{l}\text { Qualitativo, } \\
\text { Revisão } \\
\text { integrativa de } \\
\text { literatura }\end{array}$ & & $\begin{array}{c}\text { Atenção, enfoque } \\
\text { criterioso dos serviços } \\
\text { de saúde e } \\
\text { capacitação. }\end{array}$ & $\begin{array}{c}\text { Prestar o cuidado de } \\
\text { maneira adequada e com } \\
\text { o menor risco para a sua } \\
\text { saúde física e psíquica. }\end{array}$ \\
\hline 5 & Lilacs & $\begin{array}{c}\text { Qualidade de } \\
\text { vida de } \\
\text { familiares/cuida } \\
\text { do res de } \\
\text { pessoas idosas } \\
\text { com alzheimer: } \\
\text { contribuição do } \\
\text { grupo de apoio. }\end{array}$ & 2020 & $\begin{array}{l}\text { Oliveira, } \\
\text { Tamires } \\
\text { Ineu et al. }\end{array}$ & $\begin{array}{c}\text { Pesquisa } \\
\text { exploratória, } \\
\text { descritiva e } \\
\text { de abordagem } \\
\text { qualitativa }\end{array}$ & & $\begin{array}{c}\text { A importância da } \\
\text { participação dos } \\
\text { familiares/cuidador es } \\
\text { em grupos de apoio, } \\
\text { uma vez que estes têm } \\
\text { o objetivo de amenizar } \\
\text { os sentimentos } \\
\text { oriundos desse } \\
\text { processo, favorecer a } \\
\text { adaptação do } \\
\text { cotidiano, bem como } \\
\text { possibilitar maior } \\
\text { conhecimento e } \\
\text { compreensão das } \\
\text { possíveis alterações e } \\
\text { intercorrências } \\
\text { diárias. }\end{array}$ & $\begin{array}{l}\text { Amparar e acolher } \\
\text { familiares/ cuidadores e } \\
\text { comunidade em geral } \\
\text { que necessitem de um } \\
\text { espaço para discussões a } \\
\text { respeito da temática DA. }\end{array}$ \\
\hline 6 & Scielo & $\begin{array}{l}\text { Interdisciplinari } \\
\text { ty of care to the } \\
\text { elderly with } \\
\text { Alzheimer's } \\
\text { disease: } \\
\text { reflection to the } \\
\text { light of the } \\
\text { theories of } \\
\text { Leininger and } \\
\text { Heller. }\end{array}$ & 2019 & $\begin{array}{l}\text { Barbosa, } \\
\text { Maria } \\
\text { Emília } \\
\text { Marcond es } \\
\text { et al. }\end{array}$ & $\begin{array}{l}\text { Estudo de } \\
\text { reflexão }\end{array}$ & & & $\begin{array}{l}\text { Preparar o cuidador para } \\
\text { exercer o cuidado com } \\
\text { mais propriedade. }\end{array}$ \\
\hline 7 & Lilacs & $\begin{array}{c}\text { Alzheimer's } \\
\text { disease and the }\end{array}$ & 2020 & $\begin{array}{c}\text { Gonçalves, } \\
\text { Fabiana }\end{array}$ & $\begin{array}{l}\text { Revisão } \\
\text { integrativa }\end{array}$ & & & $\begin{array}{l}\text { Desenvolver estratégias } \\
\text { de cuidado para pessoas }\end{array}$ \\
\hline
\end{tabular}


Research, Society and Development, v. 11, n. 3, e1611326083, 2022

(CC BY 4.0) | ISSN 2525-3409 | DOI: http://dx.doi.org/10.33448/rsd-v11i3.26083

\begin{tabular}{|c|c|c|c|c|c|c|c|c|}
\hline & & $\begin{array}{l}\text { challenges of } \\
\text { nursing care } \\
\text { towards elderly } \\
\text { people and their } \\
\text { family } \\
\text { caregivers. }\end{array}$ & & $\begin{array}{l}\text { Cristina } \\
\text { Alves et al. }\end{array}$ & & & & $\begin{array}{c}\text { com Doença de } \\
\text { Alzheimer e seus } \\
\text { cuidadores familiars, } \\
\text { garantindo a todos } \\
\text { suporte } \\
\text { necessário. }\end{array}$ \\
\hline 8 & Scielo & $\begin{array}{l}\text { Vulnerabilidade } \\
\text { clínica e } \\
\text { funcional de } \\
\text { idosos } \\
\text { cuidadores de } \\
\text { idosos com } \\
\text { doença de } \\
\text { Alzheimer. }\end{array}$ & 2019 & $\begin{array}{l}\text { Barbosa, } \\
\text { Maria } \\
\text { Emília } \\
\text { Marcondes } \\
\text { et al. }\end{array}$ & $\begin{array}{l}\text { Estudo } \\
\text { transversal }\end{array}$ & $\begin{array}{l}\text { Incapacidade de tomar banho } \\
\text { sozinho, esquecimento; } \\
\text { desânimo, tristeza ou } \\
\text { desesperança; perda de interesse } \\
\text { por atividades prazerosas; } \\
\text { incapacidade de elevar membro } \\
\text { superior; incapacidade de segurar } \\
\text { pequenos objetos; perda de peso } \\
\text { não intencional; dificuldade para } \\
\text { caminhar; quedas frequentes; } \\
\text { perda involuntária de urina ou } \\
\text { fezes; problemas de visão e } \\
\text { audição; uso de cinco ou mais } \\
\text { medicamentos } \\
\text { diferentes. }\end{array}$ & & \\
\hline 9 & Scielo & $\begin{array}{c}\text { Desafios e } \\
\text { tecnologias de } \\
\text { cuidado } \\
\text { desenvolvidos } \\
\text { por cuidadores } \\
\text { de pacientes } \\
\text { com doença de } \\
\text { Alzheimer. }\end{array}$ & 2018 & $\begin{array}{l}\text { Schmidt, } \\
\text { Melanie, } \\
\text { Scheineider } \\
\quad \text { et al. }\end{array}$ & $\begin{array}{c}\text { Pesquisa } \\
\text { exploratória, } \\
\text { de abordagem } \\
\text { qualitativa }\end{array}$ & $\begin{array}{l}\text { Dificuldades na manutenção do } \\
\text { sono regular e tranquilo, além de } \\
\text { dificuldades ou para iniciar o } \\
\text { sono ou para despertar precoce; } \\
\text { agitação ao anoitecer. A maioria } \\
\text { dos idosos são resistentes ao } \\
\text { banho e apresentam dificuldades } \\
\text { em vestir a roupa adequada } \\
\text { conforme as estações do ano, } \\
\text { principalmente por esquecimento } \\
\text { das etapas desses procedimentos. } \\
\text { Na higiene oral a maior } \\
\text { dificuldade encontrada é o } \\
\text { cerramento dos lábios pelos } \\
\text { idosos; dificuldades de } \\
\text { mastigação e/ou deglutição; } \\
\text { incapacidade de realizar as } \\
\text { AIVD, a perda recorrente de } \\
\text { memória, da sensibilidade e } \\
\text { principalmente do juízo de valor; } \\
\text { dificuldade de aceitação e } \\
\text { deglutição das medicações e os } \\
\text { aspectos relacionados à } \\
\text { mobilidade física. }\end{array}$ & & $\begin{array}{l}\text { Prevenção de declínio } \\
\text { funcional dos cuidadores } \\
\text { idosos. }\end{array}$ \\
\hline 10 & Lilacs & $\begin{array}{c}\text { The family } \\
\text { caregiver's } \\
\text { discourse on the } \\
\text { hospitalization } \\
\text { of the elderly } \\
\text { with } \\
\text { Alzheimer's } \\
\text { disease. }\end{array}$ & 2017 & $\begin{array}{l}\text { Grillo, } \\
\text { Gabriela } \\
\text { Pizelli } \\
\text { Mocco et } \\
\text { al. }\end{array}$ & $\begin{array}{l}\text { Estudo } \\
\text { descritivo, } \\
\text { exploratório, } \\
\text { de natureza } \\
\text { qualitativa }\end{array}$ & $\begin{array}{l}\text { Confusão mental; Depressão; } \\
\text { memória recente afetada; } \\
\text { comprometimento de funções } \\
\text { cognitivas. }\end{array}$ & $\begin{array}{c}\text { Orientações ou } \\
\text { educação em saúde, } \\
\text { porque quando as } \\
\text { dúvidas, inseguranças } \\
\text { e medos são curados } \\
\text { sentem-se acolhidos e } \\
\text { seguros no processo de } \\
\text { cuidado. } \\
\end{array}$ & $\begin{array}{l}\text { Treinamento e/ou } \\
\text { especialização para } \\
\text { atender a clientele de } \\
\text { idosos e sua família. }\end{array}$ \\
\hline 11 & Scielo & $\begin{array}{c}\text { Estresse e } \\
\text { qualidade de } \\
\text { vida do } \\
\text { cuidador } \\
\text { familiar de } \\
\text { idoso portador } \\
\text { da doença de } \\
\text { Alzheimer. }\end{array}$ & 2017 & $\begin{array}{l}\text { Cesário, } \\
\text { Vanovya } \\
\text { Alves } \\
\text { Claudino et } \\
\text { al. }\end{array}$ & $\begin{array}{c}\text { Estudo } \\
\text { qualitativo do } \\
\text { tipo } \\
\text { descritivo }\end{array}$ & & $\begin{array}{c}\text { Os cuidadores } \\
\text { familiares necessitam } \\
\text { de assistência e } \\
\text { orientação das equipes } \\
\text { de saúde da família, e } \\
\text { grupo de autoajuda } \\
\text { para favorecer a } \\
\text { melhora da qualidade } \\
\text { de vida. }\end{array}$ & $\begin{array}{l}\text { Favorecer uma melhor } \\
\text { qualidade de vida para o } \\
\text { cuidador e para o idoso, } \\
\text { e consequentemente } \\
\text { presta uma melhor } \\
\text { assistência. }\end{array}$ \\
\hline 12 & Lilacs & $\begin{array}{l}\text { Vivências de } \\
\text { Cuidadores } \\
\text { Familiares de } \\
\text { Pessoas Idosas } \\
\text { com Doença de } \\
\text { Alzheimer. }\end{array}$ & 2017 & $\begin{array}{l}\text { Faria, Érica } \\
\text { Beatriz } \\
\text { Alves et al. }\end{array}$ & $\begin{array}{c}\text { Pesquisa } \\
\text { exploratória e } \\
\text { descritiva, } \\
\text { com } \\
\text { abordagem } \\
\text { qualitativa }\end{array}$ & $\begin{array}{l}\text { Distúrbios de comportamento, } \\
\text { manifestos por agitação, } \\
\text { perambulação, agressão verbal } \\
\text { e/ou física, perda de memória, } \\
\text { alteração de personalidade, } \\
\text { alucinações, distúrbios do sono e } \\
\text { violência repentina. }\end{array}$ & $\begin{array}{l}\text { Reorganização de rede } \\
\text { interativa entre os } \\
\text { familiares e a equipe } \\
\text { interdisciplinar, apoio } \\
\text { da equipe na } \\
\text { superação do trauma } \\
\text { psíquico. }\end{array}$ & $\begin{array}{c}\text { Compreender as } \\
\text { limitações e criar novas } \\
\text { estratégias voltadas às } \\
\text { necessidades dos idosos, } \\
\text { planejar intervenções que } \\
\text { visem melhorar a } \\
\text { qualidade de vida dos } \\
\text { seus cuidadores. }\end{array}$ \\
\hline 13 & Lilacs & $\begin{array}{l}\text { Personalidade } \\
\text { de cuidadores } \\
\text { de idosos com } \\
\text { demência de } \\
\text { Alzheimer e } \\
\text { funcionalidade } \\
\text { familiar: } \\
\text { contribuições } \\
\end{array}$ & 2017 & $\begin{array}{c}\text { Cruz, } \\
\text { Thiara } \\
\text { Joanna, } \\
\text { Peçanha et } \\
\text { al. }\end{array}$ & $\begin{array}{c}\text { Estudo } \\
\text { analítico, } \\
\text { observacional } \\
\text { do tipo } \\
\text { seccional. }\end{array}$ & $\begin{array}{l}\text { Perda de memoria, linguagem e } \\
\text { demais funções cognitivas. }\end{array}$ & $\begin{array}{l}\text { Suporte profissional de } \\
\text { saúde, organização da } \\
\text { família para adaptasse, } \\
\text { informações sobre a } \\
\text { doença, atenção } \\
\text { medica pessoal e apoio } \\
\text { emocional. }\end{array}$ & $\begin{array}{c}\text { Desenvolver uma } \\
\text { sistematização que } \\
\text { compreenda os cuidados } \\
\text { diário ao idoso, } \\
\text { contribuir para qualidade } \\
\text { de vida dos cuidadores e } \\
\text { orientações sobre os } \\
\text { enfrentamentos do } \\
\end{array}$ \\
\hline
\end{tabular}




\begin{tabular}{|c|c|c|c|c|c|c|c|c|}
\hline & & $\begin{array}{c}\text { para a prática de } \\
\text { enfermagem. }\end{array}$ & & & & & & cotidiano. \\
\hline 14 & Scielo & $\begin{array}{l}\text { Doença de } \\
\text { Alzheimer: } \\
\text { desafios } \\
\text { enfrentados } \\
\text { pelo cuidador } \\
\text { no cotidiano } \\
\text { familiar. }\end{array}$ & 2016 & $\begin{array}{l}\text { Kucmanski, } \\
\text { Luciane } \\
\text { Salete et al. }\end{array}$ & $\begin{array}{l}\text { Abordagem } \\
\text { qualitativa de } \\
\text { caráter } \\
\text { descritivo. }\end{array}$ & $\begin{array}{l}\text { Perda mais acentuada de } \\
\text { memória, incapacidade de } \\
\text { realizar tarefas complexas, } \\
\text { comprometimento acentuado da } \\
\text { capacidade funcional, dificuldade } \\
\text { para se alimentar, vestir-se e } \\
\text { banhar-se. }\end{array}$ & $\begin{array}{l}\text { Atenção e zelo por } \\
\text { parte dos profissionais } \\
\text { de saúde e das } \\
\text { políticas públicas, } \\
\text { receber orientações } \\
\text { sobre a evolução da } \\
\text { doença e apoio } \\
\text { emocional. }\end{array}$ & \\
\hline 15 & Scielo & $\begin{array}{c}\text { Mudanças de } \\
\text { comportamento } \\
\text { em idosos com } \\
\text { doença de } \\
\text { Alzheimer e } \\
\text { sobrecarga para } \\
\text { o cuidador. }\end{array}$ & 2016 & $\begin{array}{c}\text { Maris, } \\
\text { Aline } \\
\text { Miranda } \\
\text { Fonseca et } \\
\quad \text { al. }\end{array}$ & $\begin{array}{l}\text { O estudo foi } \\
\text { conduzido à } \\
\text { luz do } \\
\text { Interacionism } \\
\text { o Simbólico } \\
\text { (IS). }\end{array}$ & $\begin{array}{l}\text { Dor, raiva, tristeza, ou até mesmo } \\
\text { algum tipo de medo. A falta de } \\
\text { habilidade das pessoas com } \\
\text { Doença de Alzheimer em } \\
\text { reconhecer ou expressar suas } \\
\text { necessidades para seus cuidadores } \\
\text { pode resultar em manifestações } \\
\text { do tipo mudança de } \\
\text { comportamento. }\end{array}$ & $\begin{array}{l}\text { Apoio emocional, } \\
\text { tempo adequado de } \\
\text { intervalo ou descanso, } \\
\text { compartilhar o cuidado } \\
\text { com outros membros } \\
\text { da família ou um } \\
\text { cuidador forma. }\end{array}$ & $\begin{array}{l}\text { Reconhecer e acolher as } \\
\text { demandas específicas } \\
\text { dos cuidadores, de forma } \\
\text { a prover um cuidado de } \\
\text { enfermagem informativo, } \\
\text { educativo e } \\
\text { personalizado que } \\
\text { reconhece e dá conta } \\
\text { também das reações de } \\
\text { atitudes da pessoa com } \\
\text { Doença de Alzheimer, } \\
\text { em particular aquelas } \\
\text { envolvendo riscos à } \\
\text { segurança. }\end{array}$ \\
\hline 16 & Scielo & $\begin{array}{l}\text { O cuidado na } \\
\text { doença de } \\
\text { Alzheimer: as } \\
\text { representações } \\
\text { sociais dos } \\
\text { cuidadores } \\
\text { familiares. }\end{array}$ & 2016 & $\begin{array}{l}\text { Mendes, } \\
\text { Cinthia } \\
\text { Figueiredo } \\
\text { Maciel et } \\
\text { al. }\end{array}$ & $\begin{array}{c}\text { Tipo } \\
\text { empírico, } \\
\text { exploratório, } \\
\text { qualiquantitat } \\
\text { ivo. }\end{array}$ & $\begin{array}{c}\text { Perturbações da memória, } \\
\text { pensamento, compreensão e } \\
\text { linguagem, deficiência das } \\
\text { habilidades cognitivas, perda de } \\
\text { controle emocional, social, da } \\
\text { motivação, apatia e depressão. }\end{array}$ & $\begin{array}{c}\text { Atenção dos } \\
\text { profissionais da saúde, } \\
\text { bem-estar físico e } \\
\text { psíquico. }\end{array}$ & $\begin{array}{l}\text { Presta assistência diária } \\
\text { ao idoso de } \\
\text { forma paciência e } \\
\text { disponibilidade para o } \\
\text { cuidado, manter } \\
\text { estabilidade emocional } \\
\text { necessária para presta à } \\
\text { assistência de qualidade. }\end{array}$ \\
\hline 17 & Scielo & $\begin{array}{l}\text { Doença de } \\
\text { Alzheimer na } \\
\text { pessoa } \\
\text { idosa/família: } \\
\text { dificuldades } \\
\text { vivenciadas e } \\
\text { estratégias de } \\
\text { cuidado. }\end{array}$ & 2016 & $\begin{array}{l}\text { Backes, } \\
\text { Dirce Stein } \\
\text { et al. }\end{array}$ & $\begin{array}{c}\text { Pesquisa } \\
\text { exploratória, } \\
\text { descritiva, de } \\
\text { abordagem } \\
\text { qualitativa. }\end{array}$ & $\begin{array}{l}\text { Manifestam confusão e perda de } \\
\text { memória, desorientação espacial, } \\
\text { dificuldade progressiva no } \\
\text { cotidiano, mudanças na } \\
\text { personalidade e na capacidade de } \\
\text { julgamento. } \\
\text { A doença evolui para a } \\
\text { incapacidade na realização das } \\
\text { atividades da vida } \\
\text { diária, além de ansiedade, } \\
\text { delírios, alucinações, agitação } \\
\text { noturna, alterações do sono, } \\
\text { dificuldades de reconhecimento } \\
\text { de amigos e familiares. O mais } \\
\text { grave estágio é caracterizado pela } \\
\text { redução acentuada do } \\
\text { vocabulário, diminuição do } \\
\text { apetite e do peso, } \\
\text { descontrole esfincteriana e } \\
\text { posicionamento fetal. }\end{array}$ & $\begin{array}{c}\text { Construção de grupos } \\
\text { coletivos que } \\
\text { proporcione } \\
\text { estratégias do } \\
\text { autocuidado. }\end{array}$ & \\
\hline 18 & Scielo & $\begin{array}{l}\text { Teoría de } \\
\text { Kristen } \\
\text { M. Swanson } \\
\text { vinculada al } \\
\text { cuidado del } \\
\text { cuidador } \\
\text { principal de } \\
\text { pacientes con } \\
\text { enfermedad de } \\
\text { Alzheimer. }\end{array}$ & 2020 & $\begin{array}{l}\text { Petterson, } \\
\text { Mabel } \\
\text { Garzon et } \\
\text { al. }\end{array}$ & $\begin{array}{c}\text { Revisão } \\
\text { bibliográfica } \\
\text { descritiva. }\end{array}$ & & $\begin{array}{c}\text { Conhecimento } \\
\text { necessário para } \\
\text { executar o cuidado, } \\
\text { bem-estar, grupos de } \\
\text { apoio para incentivar o } \\
\text { autocuidado e } \\
\text { envolver outros } \\
\text { familiares no } \\
\text { desempenho das } \\
\text { funções. }\end{array}$ & $\begin{array}{c}\text { Adquirir os } \\
\text { conhecimentos e } \\
\text { habilidades necessários, } \\
\text { protegendo e } \\
\text { preservando a dignidade } \\
\text { humana dos outros. }\end{array}$ \\
\hline 19 & Scielo & $\begin{array}{l}\text { Gerontotecnolo } \\
\text { gias utilizadas } \\
\text { pelos } \\
\text { familiares/cuida } \\
\text { dor es de idosos } \\
\text { com Alzheimer: } \\
\text { contribuição ao } \\
\text { cuidado } \\
\text { complexo. }\end{array}$ & 2018 & $\begin{array}{c}\text { Ilha, } \\
\text { Silomar et } \\
\text { al. }\end{array}$ & $\begin{array}{c}\text { Estudo } \\
\text { exploratório, } \\
\text { descritiva, de } \\
\text { abordagem } \\
\text { qualitativa. }\end{array}$ & $\begin{array}{l}\text { Dificuldade de reconhecer locais } \\
\text { comuns, como o próprio } \\
\text { domicílio; esquecimento de } \\
\text { caminhos e destinos; não } \\
\text { aceitação do banho; repetição de } \\
\text { assuntos e a irritabilidade; } \\
\text { incapacidade de reconhecer o } \\
\text { valor do } \\
\text { dinheiro. }\end{array}$ & & $\begin{array}{c}\text { Socializar as } \\
\text { gerontotecnologias que } \\
\text { podem contribuir } \\
\text { diretamente no cuidado, } \\
\text { possibilitando melhorar o } \\
\text { bem-estar das pessoas } \\
\text { idosas para que } \\
\text { vivenciem o processo da } \\
\text { DA com maior segurança } \\
\text { física e QV. }\end{array}$ \\
\hline
\end{tabular}

Fonte: Autoria própria (2021).

A Teoria do Alcance de Metas proposta por Imogene King apresenta um relevante referencial teórico no agir em 
Enfermagem, pois, através das metas estabelecidas, o cuidado é direcionado para a valorização da tomada de decisão compartilhada entre enfermeiro e paciente. Para King, o indivíduo é formado por três sistemas interativos: pessoal, interpessoal e social, que são abertos ao ambiente de modo que possam interagir para garantir seu equilíbrio e saúde (Pissinati et al., 2020).

Ainda de acordo com Pissinati et al. (2020), referenciando King, o sistema pessoal corresponde as características pessoais de cada indivíduo, percebendo suas concepções, expectativas e ansiedades diante de eventos vivenciados. O sistema interpessoal interpreta as interações humanas, define como um indivíduo reage diante das ações de outro, ainda abrangendo a comunicação, estresse, papel e transação. Já o sistema social é visualizado quando há o encontro entre equipes com interesses e necessidades especiais, representa os papéis, comportamentos e práticas que delimitam as ações do grupo.

A fim de compreender a contribuição da Teoria do Alcance de Metas na construção de uma assistência efetiva ao cuidador familiar do idoso portador de Alzheimer, buscou- se organizar os resultados em três categorias, quais sejam: Aspectos do comportamento do idoso portador de Doença de Alzheimer; Necessidades do cuidador familiar do idoso; Metas a serem estabelecidas na prestação de assistência ao cuidador familiar do idoso com Alzheimer.

\subsection{Aspectos do comportamento do idoso portador de Doença de Alzheimer}

Diversos aspectos podem ingressar no comportamento do idoso portador de Doençade Alzheimer. De acordo com Delfino et al. (2021) o idoso apresenta doze sintomas neuropsiquiátricos, sendo apatia, ansiedade e depressão os sintomas mais frequentes. Associaram-se alguns desses sintomas a sobrecarga do cuidador. Barbosa et al. (2019) traz aspectos como incapacidade de tomar banho sozinho, em vestir a roupa, desânimo, tristeza ou desesperança, perda de interesse por atividades prazerosas e perda involuntária de urina ou fezes.

A doença pode evoluir para a incapacidade na realização das atividades de vida diária, além de manifestar ansiedade, delírios, alucinações, agitação noturna, alterações do sono e dificuldades de reconhecimento de amigos e familiares, redução acentuada do vocabulário, diminuição do apetite e do peso, descontrole esfincteriano e posicionamento fetal e dificuldade de reconhecer locais comuns (Backes et al., 2016).

Estudos como o de Grillo et al. (2017) e Cruz et al. (2017) apontam para perda de memória, linguagem, funções cognitivas, controle emocional, social, perda da motivação e depressão. De acordo com Schmidt et al. (2018) o idoso pode apresentar dificuldades de mastigação e/ou deglutição, manutenção do sono regular e tranquilo, além de dificuldades ou para iniciar o sono ou para despertar precoce, agitação ao anoitecer, apresenta também incapacidade de realizar as AVD e dificuldades em vestir roupas adequadas conforme as estações do ano.

É possível observar, portanto, que todos esses aspectos estão associados à sobrecarga do cuidador.

\subsection{Necessidades do cuidador familiar do idoso}

O cuidador é submetido a uma jornada diária assídua, já que irá acompanhar o idoso em todas as atividades diárias e acaba necessitando de cuidados. De acordo com Delfino et al. (2021) esse cuidador deve receber orientação e cuidado da equipe de saúde da família. Já Dadalto et al. (2021) apontam que é necessário reunir várias estratégias terapêuticas para capacitação de competências desses profissionais, encontros por meio de grupos focais, que haja possibilidade de acompanhamento sistemático e longitudinal para os cuidadores familiares.

Além de necessitar de orientação do grupo de saúde também é importante a participação dos familiares/cuidadores em grupos de apoio, para amenizar os sentimentos ruins que possam apresentar durante processo, podendo favorecer a adaptação no cotidiano e possibilite adquirir conhecimento e compreensão de todas as etapas que podem afetar a vida do idoso e consequentemente concernir na vida do cuidador familiar (Oliveira et al., 2020).

Na perspectiva de Grillo et al. (2017) e Cesário et al. (2017), os cuidadores necessitam não só de orientações, mas também educação em saúde para sanar as dúvidas, inseguranças e ajudar a curar medos. Grupos de autoajuda podem contribuir 
para que eles sintam-se acolhidos e seguros e consequentemente melhorem sua qualidade de vida. Faria et al. (2017) ressaltam a reorganização de rede interativa entre os cuidadores familiares e a equipe interdisciplinar para fornecer toda a assistência aos traumas psíquicos.

Cruz et al. (2017) apontam que esses cuidadores necessitam de suporte profissional de saúde, organização da família, atenção médica pessoal e apoio emocional. Além de precisar do suporte profissional, de acordo com Maris et al. (2016) e Kucmanski et al. (2016) necessitam de atenção e zelo por parte das políticas públicas, um tempo adequado de intervalo ou descanso e o compartilhamento do cuidado com outros membros da família ou um cuidador formal.

\subsection{Metas a serem estabelecidas na prestação de assistência ao cuidador familiar doidoso com Alzheimer}

Segundo Maris et al. (2016), para prestar assistência, deve-se conhecer e acolher as demandas específicas dos cuidadores, assim provendo um cuidado informativo e educativo, dar conta também das reações e atitudes desenvolvidas por pessoa com Doença de Alzheimer e consequentemente prestar assistência de qualidade visto que eles podem apresentar riscos à sua própria segurança.

É importante incentivar o bem-estar próprio do cuidador no pós-alta para garantir e melhorar o acesso aos cuidados de saúde, consequentemente atender as necessidades que devem ser atribuídas no cuidado do idoso portador de Alzheimer, oferecendo orientações sobre o cuidado de maneira adequada e com menor risco, assim garantindo segurança para a sua saúde física e psíquica (Damásio et al., 2020; Santana et al., 2020).

Em relação às metas que devem ser estabelecidas para prestar uma assistência de qualidade, Ilha et al. (2016) destacam a utilização das gerontotecnologias, que é direcionada para contribuir diretamente no cuidado, possibilitando melhorar a assistência das pessoas portadoras da doença de Alzheimer e promover uma boa vivência durante todo o progresso dadoença.

De acordo com Schmidt et al. (2018), a prevenção de declínio funcional dos cuidadores idosos seriam as tecnologias de cuidado adaptadas para o ambiente domiciliar, objetivando a segurança física do idoso com Alzheimer com o uso de colchões adequados, musicoterapia e atividades de lazer, proporcionando bem-estar ao cuidador. Cesáreo et al. (2017) referem que a distribuição de atribuições entre os familiares de idosos com Alzheimer evita a responsabilidade em um único familiar e pode favorecer uma melhor qualidade de vidapara os mesmos.

Segundo Faria et al. (2017), a contribuição do enfermeiro da estratégia de saúde da família é importante pois pode criar novas estratégias de cuidado para os idosos portadores de Alzheimer e seus cuidadores, além de facilitar a compreensão das limitações destes e planejar intervenções que visem melhorar a qualidade de vida. Já Kucmanski et al. (2016) observaram que a informação oferecida aos cuidadores pelos enfermeiros diminui a ansiedade e insegurança em relação aos cuidados, refletindo em maior capacidade e disponibilidade para exercer o cuidado.

\section{Conclusão}

Os resultados desse estudo levam à identificação dos aspectos comportamentais do idoso portador da Doença de Alzheimer, às necessidades do cuidador familiar e aoestabelecimento de metas de acordo com a Teoria de King para a prestação da assistência. O cuidador familiar possui um destaque na assistência ao idoso portador de Alzheimer, visto queé aquele que possui o maior contato com o paciente, portanto, necessita de orientações, educação em saúde e saber reconhecer e identificar seus limites.

Neste sentido, a Teoria do Alcance de Metas proposta por Imogene King contribui para a construção de uma assistência efetiva ao cuidador familiar do idoso portador de Alzheimer, à medida que permite compreender o impacto do cuidado ao paciente sobre esse cuidador, suas necessidades pessoais, bem como as estratégias terapêuticas que podem ser 
utilizadas a fim de alcançar as metas estabelecidas e evitar a sobrecarga do cuidado.

Ainda que se tenha obtido uma amostra de 19 artigos, foram poucos os estudosencontrados que enfatizam a saúde do cuidador familiar, em comparação aos artigos com temas voltados à saúde do idoso portador de Alzheimer, o que demonstra uma lacuna que precisa ser trabalhada. Sugere-se, portanto, que mais pesquisas sejam realizadas com o olhar direcionado para o cuidador familiar.

\section{Referências}

Barbosa, M. E. M., Corso, E. R., Scolari, G. A. S. \& Carreira, L. (2019). Interdisciplinarity of care to the elderly with Alzheimer's disease: reflection to the light of the theories of Leininger and Heller. Esc. Anna Nery, 24(1), 1-8.

Barbosa, M. E. M., Bertelli, E. V. M., Scolari, G. A. S., Bortolanza, M. C. Z., Higarashi, I. H., \& Carreira, L. (2021). Vulnerabilidade clínica e funcional de idosos cuidadores de idosos com doença de Alzheimer. Esc. Anna Nery, 1-7.

Cesário, V. A. C., Leal, M. C. C., Marques, A. P. O., \& Claudio, K. A. (2017). Estresse e qualidade de vida do cuidador familiar de idoso portadorda doença de Alzheimer. Saúde Debate., 41 (112), 171-182.

Cruz, T. J. (2017). Personalidade de cuidadores de idosos com demência de Alzheimer efuncionalidade familiar: contribuições para a prática de enfermagem. Universidade doEstado do Rio de Janeiro.

Dadalto, E. V., \& Cavalcante, E. V. (2021). O lugar do cuidador familiar de idosos com Doença de Alzheimer: uma revisão integrativa de literatura no Brasil e Estados Unidos. Ciências e Saúde Coletiva, 147-157.

Damásio, C. S. R., Oliveira, A. D. S., Santos, A. M. R., \& Almeida, C. A. P. L. (2020). Fatores associados à qualidade de vida na perspectiva do cuidador de idosos com doença de Alzheimer. Sociedade e Saúde, 36(2), 652-662.

Delfino, L. L., Komatsu, R. S., Kamatsu, C., Neri, A. L., \& Cachioni, M. (2021). Sintomas neuropsiquiátricos associados à sobrecarga e depressão do cuidador familiar. Dement Neuropsychol, 128-135.

Faria, E. B. A., Scardoelli, M. G. C., Castro, V. C., \& Nishida, F. S. (2017). Vivências de Cuidadores Familiares de Pessoas Idosas com Doença deAlzheimer. Ciência, cuidado e saúde, (16).

Gonçalves, F. C., \& Lima, I. C. S. (2020). Alzheimer's disease and the challenges of nursing care towards elderly people and their family caregivers. Revista Online de Pesquisa, (12), 1274- 1282.

Grillo, G. L., Marins, A. M. F., \& Mello, R. (2017). The family caregiver's discourse on the hospitalization of the elderly with Alzheimer's disease. Revista de Pesquisa Cuidado é Fundamental Online, (94), 1068-1073.

Ilha, S., Backes, D. S., Santos, S. S. C., Abreu, D. P. G., Silva, B. T., \& Pelzer, M. T. (2016). Doença de Alzheimer na pessoa idosa/família: dificuldades vivenciadas eestratégias de cuidado. Esc Anna Nery. 138-146.

Ilha, S., Santos, S. S. C., Backes, D. S., Barros., E. J. L., Pelzer, M. T., \& Abreu, D. P. G. (2018). Gerontotecnologias utilizadas pelos familiares/cuidadores de idosos com alzheimer: contribuição ao cuidado complexo. Texto e Contexto, 1- 11.

Kucmanski, L.S. Zenevicz, L., Geremia, D. S., Madureira, V. S, F., Silva, T. F., \& Souza, S. S. (2016). Doença de Azheimer: desafios enfrentados pelo cuidador no cotidiano familiar. Rev. bras. geriatr. gerontol. 19(6), 1022-1029.

Maris, A. M. F., Hansel, C. G., \& Silva, J. (2016). Mudanças de comportamento em idosos com doença de alzheimer esobrecarga para o cuidador. Esc Anna Nery, 352- 356 .

Mendes, C. F. M., \& Santos, A. L. S. (2016). O cuidado na doença de Alzheimer: as representações sociais dos cuidadores familiares. Saúde e Sociedade, 25 (1), 121- 132 .

Oliveira, T. I., Maziero, B. R., Buriol, D., Rosa, P. H., \& Ilha, S. (2020). Qualidade de vida de familiares/cuidadores de pessoas idosas com alzheimer: contribuição do grupo de apoio. Revista de Pesquisa Cuidado é Fundamental, 12.7568, 828- 832.

Petterson, M. G., Medina, R. I., Cuesta, Y. P., Pérez, N. O. B., \& Jiménez, M. R. (2020). Teoría de Kristen M. Swanson vinculada al cuidado del cuidador principal de pacientes con enfermedad de Alzheimer. Revista Cubana de Enfermería, 36 (4).

Pissinati, P. S. C., Martins, E. A. P., Costa, R. C., \& Haddad, M. C. F. L. (2020). Estabelecimento de metas no planejamento da aposentadoria: reflexão à luz de Imogene King. Revista Mineira de Enfermagem, 24, 1-4.

Santana, R. F., Soares, T. S., Santos, C. T. B., Hercules, A. B. S., Lindolpho, M. C., \& Boechat, Y. E. M. ( 2020). Telecuidado no acompanhamento pós-alta de idosos com demência e seus cuidadores: quase-experimental. Online Brazilian Journal of Nursing, .19 (2).

Schmidt, M. S., Lochs,M. O. H., Hammershimidt, K. S. A., Fernades, D. L. R., Tristão, F. R., \& Girondi, J. B. R. (2018). Desafios e tecnologias de cuidado desenvolvidos por cuidadores de pacientes com doença de Alzheimer. Revista Brasileira de Geriatria e Gerontologia, $601-609$

Silva, N. R. F., Farias, D. C., Sousa, J. R., Bezerra, F. M. C., Ferreira, L. S., \& Carvalho, P. M. G. (2018). Teorias de Enfermagem Aplicadas no Cuidado A Pacientes Oncológicos:Contribuição Para Prática Clínica do Enfermeiro. Revista UNINGÁ, 55 (2), 59 - 71. 